\title{
SOME REMARKS \\ ON A QUASI-STEADY-STATE APPROXIMATION OF THE NAVIER-STOKES EQUATION
}

\author{
JOHN R. CANNON ${ }^{1}$ AND GEORGE H. KNIGHTLY ${ }^{2}$
}

(Received September 17, 1986; revised August 2, 1987)

\begin{abstract}
A quasi-steady-state approximation to the Navier-Stokes equation is the corresponding equation with nonhomogeneous forcing term $f(x, t)$, but with the term $v_{t}$ deleted. For solutions that are zero on the boundary, the difference $\mathrm{z}$ between the solution of the Navier-Stokes equation and the solution of this quasi-steadystate approximation is estimated in the $L_{2}$ norm $\|z\|$ with respect to the spatial variables. For sufficiently large viscosity or sufficiently small body force $f$, the inequality

$$
\|\mathbf{z}(\cdot, t)\|^{2} \leq\|\mathbf{z}(\cdot, 0)\|^{2} \exp \{-\beta t\}+C \sup _{0<t<T}\left\|\mathbf{f}_{t}\right\|
$$

holds for $0<t \leq T$ and certain real numbers $C, \beta>0$.
\end{abstract}

\section{Introduction}

For fluid problems governed by systems of partial differential equations of NavierStokes type, the theory for boundary value problems and the steady equations is generally more complete than that for the corresponding initial boundary value problems and the time-dependent equations. For example, see the books of Ladyzhenskaya [4], Shinbrot [5] and Temam [6] for treatment of the wellposedness of problems involving the Navier-Stokes equation. One situation in which the present theory of the unsteady equations is markedly less developed than that for the steady equations occurs in [1] and [2], where convective Stefan problems governed by Boussinesq systems (see, e.g., Joseph [3]) are investigated. In [1], the existence of a weak solution was proved for a nonlinear stationary

\footnotetext{
${ }^{1}$ Washington State University, Pullman, Washingtom 99164, U.S.A.

${ }^{2}$ University of Massachusetts, Amherst, Massachusetts 01003, U.S.A.

(C) Copyright Australian Mathematical Society 1988, Serial-fee code 0334-2700/88
} 
free-boundary problem describing the motion, freezing and thawing of a mixture of ice and water occupying a region of three-dimensional space. The associated non-stationary initial boundary value problem was studied in [2]; however the existence result there was restricted to two space dimensions, and convection was limited to the temperature equation.

Such limitations lead to the question of whether intermediate problems can be found allowing some time dependence and offering some of the advantages of the steady theory, while yielding a reasonable approximation to the fully nonstationary problem. The class of problems studied here concerns the Navier-Stokes equation for a viscous incompressible fluid, when the motion is nearly steady, with slow variations in time due to external force variations. We drop the time derivative from the equations and apply the term "quasi-steady-state" to the resulting equations and problems. Here we begin a study of quasi-steady-state matters by investigating whether the solution of a quasi-steady-state problem offers a good approximation to the solution of the corresponding fully nonstationary Navier-Stokes problem. We show that the difference between the two solutions remains small if it is initially small, if the time derivative of the external force is small and if a certain parameter $P$ (see Section 4), similar to a Reynolds number, is small.

\section{Formulation of the problem and results}

In this section we describe the quasi-steady-state approximation, establish the setting for our work and state the approximation theorem.

Let $x=\left(x_{1}, \ldots, x_{n}\right)$ denote a point in Euclidean space $\mathbf{R}^{n}, n=2,3$, and let $\Omega$ denote a bounded domain in $\mathbf{R}^{n}$ with boundary $\partial \Omega$. For $T>0$, we define $Q_{T}=\Omega \times(0, T], S_{T}=\partial \Omega \times(0, T]$ and $\bar{Q}_{T}=(\Omega \cup \partial \Omega) \times[0, T]=\bar{\Omega} \times[0, T]$. Let $\mathbf{v}: \bar{Q}_{T} \rightarrow \mathbf{R}^{n}$ denote the solution velocity of the initial boundary value problem for the Navier-Stokes equation

$$
\mathbf{v}_{t}+\mathbf{v} \cdot \nabla \mathbf{v}+\nabla p=\nu \Delta \mathbf{v}+\mathbf{f} \text { in } Q_{T}
$$

and continuity equation

$$
\operatorname{div} \mathbf{v}=0 \quad \text { in } Q_{T},
$$

subject to boundary condition

$$
\mathbf{v}=\mathbf{0} \text { on } S_{T}
$$

and initial condition

$$
\mathbf{v}(x, 0)=\mathbf{a}(x), \quad x \in \Omega,
$$


and let $\mathbf{w}: \bar{Q}_{T} \rightarrow \mathbf{R}^{n}$ denote the solution velocity of the following quasi-stationary problem

$$
\begin{gathered}
\mathbf{w} \cdot \nabla \mathbf{w}+\nabla q=\nu \Delta \mathbf{w}+\mathbf{f} \text { in } Q_{T} \\
\operatorname{div} \mathbf{w}=\mathbf{0} \text { in } Q_{T}, \\
\mathbf{w}=0 \text { on } S_{T},
\end{gathered}
$$

where $\mathbf{v}_{t} \equiv \partial \mathbf{v} / \partial t, \nabla$ denotes the gradient with respect to the spatial variables $x_{i}, i=1, \ldots, n, \Delta$ denotes the Laplacian with respect to the spatial variables, $\operatorname{div} \mathbf{v}=\boldsymbol{\nabla} \cdot \mathbf{v}, p$ and $q$ denote scalar-valued functions representing the pressures of the fluid, $\mathbf{f}=\mathbf{f}(x, t)$ is a vector-valued function which represents the body forces, $\mathbf{a}=\mathbf{a}(x)$ satisfies $\operatorname{div} \mathbf{a}=0$, and the scalar $\nu>0$ denotes the viscosity.

In this article we shall study the difference $z=v-w$, which satisfies the system

$$
\begin{gathered}
\mathbf{z}_{t}+\mathbf{z} \cdot \boldsymbol{\nabla} \mathbf{z}+\mathbf{w} \cdot \boldsymbol{\nabla} \mathbf{z}+\mathbf{z} \cdot \nabla \mathbf{w}+\nabla h=\nu \Delta \mathbf{z}-\mathbf{w}_{t} \text { in } Q_{T}, \\
\operatorname{div} \mathbf{z}=0, \quad \text { in } Q_{T}, \\
\quad \mathbf{z}=0, \quad \text { on } S_{T}, \\
\mathbf{z}(x, 0)=\mathbf{a}(x)-\mathbf{w}(x, o), \quad x \in \Omega,
\end{gathered}
$$

where $h=p-q$ denotes the pressure difference. Before stating the approximation theorem it is convenient to recall some well-known facts and introduce some additional notation.

For square-integrable vector fields $\mathbf{g}: \Omega \rightarrow \mathbf{R}^{n}$, let

$$
\|g\|^{2}=\int_{\Omega} \mathbf{g} \cdot \mathbf{g} d x
$$

If, in addition, $\mathbf{g}$ is smooth with compact support in $\Omega$ we write

$$
\begin{gathered}
\nabla \mathbf{g}: \nabla \mathbf{g}=\sum_{i, k=1}^{n}\left(\partial g_{i} / \partial x_{k}\right)^{2}, \\
\|\nabla \mathbf{g}\|_{:}^{2}=\int_{\Omega} \nabla \mathbf{g}: \nabla \mathbf{g} d x
\end{gathered}
$$

and denote by $\dot{W}_{2}^{1}(\Omega)$ the closure of such vector fields in the norm $\left\{\|g\|^{2}+\right.$ $\left.\|\nabla \boldsymbol{g}\|^{2}\right\}^{1 / 2}$.

Define, for $\mathbf{w}(x, t)$ and $\mathbf{z}(x, t)$ satisfying (2) and (3),

$$
\phi^{2}(t)=\int_{\Omega} \nabla \mathbf{z}: \nabla \mathbf{z} d x
$$

and

$$
\tilde{\phi}^{2}(t)=\int_{\Omega} \nabla \mathbf{w}: \nabla \mathbf{w} d x
$$


From Lemmas 1 and 2 of $\left[4\right.$, pp. 8-9] and their application for $\mathrm{z} \in \mathscr{W}_{2}^{1}(\Omega)$, there follows

$$
\int_{\Omega}|\mathbf{z}|^{4} d x=\int_{\Omega}\left(\sum_{i=1}^{n} z_{i}^{2}\right)^{2} d x \leq \begin{cases}2\|\mathbf{z}\|^{2} \phi^{2}(t), & \text { if } n=2 \\ 4\|z\| \phi^{3}(t), & \text { if } n=3 .\end{cases}
$$

By an application of the Schwarz inequality for vectors and integrals, together with (7), we obtain

$$
\begin{aligned}
\left|\int_{\Omega}(\mathbf{z} \cdot \nabla \mathbf{w}) \cdot \mathbf{z} d x\right| & \leq\left(\int_{\Omega}|\mathbf{z}|^{4} d x\right)^{1 / 2} \tilde{\phi}(t) \\
& \leq \begin{cases}\sqrt{2}\|\mathbf{z}\| \phi(t) \tilde{\phi}(t), & \text { if } n=2 \\
2\|\mathbf{z}\|^{1 / 2} \phi^{3 / 2}(t) \tilde{\phi}(t), & \text { if } n=3 .\end{cases}
\end{aligned}
$$

Let $\mu_{1}$ denote the smallest positive eigenvalue of $-\Delta$ in $\Omega$ with zero boundary conditions. Then the Poincaré inequality $\left[4\right.$, p. 11] for $g \in \stackrel{\circ}{W}_{2}^{1}(\Omega)$ is

$$
\|\mathbf{g}\|^{2} \leq \frac{1}{\mu_{1}}\|\nabla \mathbf{g}\|^{2}
$$

From (8) and (9) we have

$$
\left|\int_{\Omega}(\mathbf{z} \cdot \nabla \mathbf{w}) \cdot \mathbf{z} d x\right| \leq c_{n} \tilde{\phi}(t) \phi^{2}(t), \quad n=2,3
$$

where

$$
c_{n}= \begin{cases}\sqrt{2} \mu_{1}^{-1 / 2}, & n=2 \\ 2 \mu_{1}^{-1 / 4}, & n=3 .\end{cases}
$$

Our principal result is contained in the following theorem.

THEOREM. Let $\mathrm{z}(x, t)$ satisfy (3), and let $\mathrm{z}$ and $\mathrm{w}$ lie in $\dot{W}_{2}^{1}(\Omega)$ for $0 \leq t \leq$ T. Then, for

$$
\begin{gathered}
\beta_{n}(t)=\mu_{1}\left(\nu-c_{n} \nu^{-1} \mu_{1}^{-1 / 2}\|\mathbf{f}\|\right)>0 \\
\|\mathbf{z}(\cdot, t)\|^{2} \leq\|\mathbf{a}(\cdot)-\mathbf{w}(\cdot, 0)\|^{2} \exp \left\{-\int_{0}^{t} \beta_{n}(\tau) d \tau\right\} \\
+\int_{0}^{t}\left(\beta_{n}(n)\right)^{-3}\left\|\mathbf{f}_{\eta}(\cdot, \eta)\right\|^{2} \exp \left\{-\int_{\eta}^{t} \beta_{n}(\tau) d \tau\right\} d \eta
\end{gathered}
$$

holds for $0 \leq t \leq T$. Moreover, for $0<\beta<\mu_{1} \nu$ and

$$
\begin{gathered}
\sup _{0<t<T}\|\mathbf{f}(\cdot, t)\| \leq c_{n}^{-1} \nu^{2} \mu_{1}^{1 / 2}\left(1-\frac{\beta}{\mu_{1} \nu}\right) \\
\|\mathbf{z}(\cdot, t)\|^{2} \leq\|\mathbf{a}(\cdot)-\mathbf{w}(\cdot, 0)\|^{2} \exp \{-\beta t\}+\beta^{-4}(1-\exp \{-\beta t\}) \sup _{0 \leq \eta \leq t}\left\|\mathrm{f}_{\eta}(\cdot, \eta)\right\|^{2}
\end{gathered}
$$

holds for $0 \leq t \leq T$. 


\section{Proof of the theorem}

The following proof of the theorem stated in Section 2 employs the methods and notation of Ladyzhenskaya [4, pp. 168-172].

PROOF. By taking the scalar product of the first partial differential equation in (3) with $\mathbf{z}(x, t)$, integrating over $\Omega$, and noting that $\operatorname{div} \mathbf{z}=\operatorname{div} \mathbf{w}=0$ implies

$$
\int_{\Omega}(\mathbf{z} \cdot \nabla \mathbf{z}) \cdot \mathbf{z} d x=\int_{\Omega}(\mathbf{w} \cdot \nabla z) \cdot \mathbf{z} d x=\int_{\Omega} z \cdot \nabla h d x=0
$$

we obtain

$$
\int_{\Omega} \mathbf{z} \cdot \mathbf{z}_{t} d x+\int_{\Omega}(\mathbf{z} \cdot \nabla \mathbf{w}) \cdot \mathbf{z} d x=\nu \int_{\Omega} \mathbf{z} \cdot(\Delta \mathbf{z}) d x-\int_{\Omega} \mathbf{w}_{t} \cdot \mathbf{z} d x .
$$

Integrating the first term on the right hand side of (17) by parts we obtain the equation

$$
\frac{1}{2} \frac{d}{d t}\|\mathbf{z}\|^{2}+\nu \phi^{2}(t)=-\int_{\Omega}(\mathbf{z} \cdot \nabla \mathbf{w}) \cdot \mathbf{z} d x-\int_{\Omega} \mathbf{w}_{t} \cdot \mathbf{z}, d x .
$$

Then, recalling (10), we see that

$$
\frac{1}{2} \frac{d}{d t}\|\mathbf{z}\|^{2}+\left(\nu-c_{n} \tilde{\phi}(t)\right) \phi^{2}(t) \leq\left\|\mathbf{w}_{t}\right\|\|\mathbf{t}\|
$$

holds via Schwarz's inequality applied to the second term on the right side of (18). From (9) and the assumption $\left(\nu-c_{n} \tilde{\phi}(t)\right)>0$, we obtain

$$
\frac{1}{2} \frac{d}{d t}\|\mathbf{z}\|^{2}+\mu_{1}\left(\nu-c_{n} \tilde{\phi}(t)\right)\|\mathbf{z}\|^{2} \leq\|\mathbf{z}\|\left\|\mathbf{w}_{t}\right\| .
$$

Hence, applying $a b \leq \varepsilon a^{2}+b^{2} / 4 \varepsilon$ with $\varepsilon=\frac{1}{2} \mu_{1}\left(\nu-c_{n} \tilde{\phi}(t)\right)$, we see that

$$
\frac{1}{2} \frac{d}{d t}\|\mathbf{z}\|^{2}+\frac{1}{2} \mu_{1}\left(\nu-c_{n} \tilde{\phi}(t)\right)\|\mathbf{z}\|^{2} \leq\left\|\mathbf{w}_{t}\right\|^{2} /\left\{2 \mu_{1}\left(\nu-c_{n} \tilde{\phi}(t)\right)\right\}
$$

Let

$$
\alpha_{n}(t)=\mu_{1}\left(\nu-c_{n} \tilde{\phi}(t)\right)
$$

Then, we have

$$
\frac{d}{d t}\|\mathrm{z}\|^{2}+\alpha_{n}(t)\|\mathrm{z}\|^{2} \leq\left(\alpha_{n}(t)\right)^{-1}\left\|\mathbf{w}_{t}\right\|^{2} .
$$

Multiplying by the integrating factor $\exp \left\{\int_{0}^{t} \alpha_{n}(\tau) d \tau\right\}$ and integrating from 0 to $t$, we see that

$$
\begin{aligned}
\|\mathrm{z}(\cdot, t)\|^{2} \leq & \|\mathbf{a}(\cdot)-\mathbf{w}(\cdot, 0)\|^{2} \exp \left\{-\int_{0}^{t} \alpha_{n}(\tau) d \tau\right\} \\
& +\int_{0}^{t}\left(\alpha_{n}(\eta)\right)^{-1}\left\|\mathbf{w}_{\eta}(\cdot, \eta)\right\|^{2} \exp \left\{-\int_{\eta}^{t} \alpha_{n}(\tau) d \tau\right\} d \eta
\end{aligned}
$$


In order to complete our study of the difference $\mathbf{z}=\mathbf{v}-\mathbf{w}$, we need estimates of $\tilde{\phi}(t)$ and $\left\|w_{t}\right\|^{2}$ in terms of the data $f$. Taking the scalar product of the first equation in (2) with $w$ and integrating over $\Omega$ we obtain

$$
\nu \tilde{\phi}^{2}(t)=\int_{\Omega} \mathbf{f} \cdot \mathbf{w} d x .
$$

Estimating the right hand side of (25) by Schwarz's inequality, we see that

$$
\nu \tilde{\phi}^{2}(t) \leq\|\mathbf{w}\|\|\mathbf{f}\| \leq \mu_{1}^{-1 / 2} \tilde{\phi}(t)\|\mathbf{f}\|
$$

via the Poincaré inequality (9). Hence,

$$
\tilde{\phi}^{2}(t) \leq \nu^{-2} \mu_{1}^{-1}\|\mathrm{f}\|^{2}
$$

and another application of (9) yields

$$
\|\mathbf{w}\|^{2} \leq\left(\nu \mu_{1}\right)^{-2}\|\mathbf{f}\|^{2} .
$$

Next we differentiate the equations in (2) with respect to $t$ to obtain

$$
\begin{gathered}
\mathbf{w}_{t} \cdot \nabla \mathbf{w}+\mathbf{w} \cdot \nabla \mathbf{w}_{t}+\nabla q_{t}=\nu \Delta \mathbf{w}_{t}+\mathbf{f}_{t} \text { in } Q_{T}, \\
\operatorname{div} \mathbf{w}_{t}=0 \quad \text { in } Q_{T}, \quad \mathbf{w}_{t}=0 \quad \text { on } S_{T} .
\end{gathered}
$$

By taking the scalar product of $w_{t}$ with the first equation in (29), integrating over $\Omega$, and using $\operatorname{div} \mathbf{w}_{t}=0$ as in (16), we find that

$$
\int_{\Omega}\left(\mathbf{w} \cdot \nabla \mathbf{w}_{t}\right) \cdot \mathbf{w}_{t} d x=\int_{\mathbf{\Omega}} \mathbf{w}_{t} \cdot \nabla q_{t} d x=0,
$$

and that

$$
\nu \tilde{\psi}^{2}(t)=-\int_{\Omega}\left(\mathbf{w}_{t} \cdot \nabla \mathbf{w}\right) \cdot \mathbf{w}_{t} d x+\int_{\Omega} \mathbf{w}_{t} \cdot \mathbf{f}_{t} d x
$$

where

$$
\tilde{\psi}^{2}(t)=\int_{\Omega} \nabla w_{t}: \nabla w_{t} d x .
$$

Proceeding as in the derivation of (10), we obtain

$$
\left|\int_{\Omega}\left(\mathbf{w}_{t} \cdot \nabla \mathbf{w}\right) \cdot \mathbf{w}_{t} d x\right| \leq c_{n} \tilde{\phi}(t) \tilde{\psi}^{2}(t) .
$$

From (9) we have

$$
\left|\int_{\Omega} \mathbf{w}_{t} \cdot \mathbf{f}_{t} d x\right| \leq \mu_{1}^{-1 / 2} \tilde{\psi}(t)\left\|\mathbf{f}_{t}\right\|
$$

Thus, we see that

$$
\nu \tilde{\psi}^{2}(t) \leq c_{n} \tilde{\phi}(t) \tilde{\psi}^{2}(t)+\mu_{1}^{-1 / 2} \tilde{\psi}(t)\left\|\mathbf{f}_{t}\right\| .
$$

applying $a b \leq \varepsilon a^{2}+b^{2} / 4 \varepsilon$, we obtain

$$
\left(\nu-c_{n} \tilde{\phi}(t)-\varepsilon \mu_{1}^{-1}\right) \tilde{\psi}^{2}(t) \leq\left\|f_{t}\right\|^{2} / 4 \varepsilon .
$$


Selecting $\varepsilon=\alpha_{n}(t) / 2$, we see that

$$
\tilde{\psi}^{2}(t) \leq \mu_{1}\left\|\mathbf{f}_{t}\right\|^{2} / \alpha_{n}^{2}(t),
$$

and from (37) it follows via the Poincaré inequality that

$$
\left\|\mathbf{w}_{t}\right\|^{2} \leq\left(\alpha_{n}(t)\right)^{-2}\left\|\mathbf{f}_{t}\right\|^{2} .
$$

We assume that

$$
\beta_{n}(t)=\mu_{1}\left(\nu-c_{n} \nu^{-1} \mu_{1}^{-1 / 2}\|\mathbf{f}\|\right)>0 .
$$

Then, from (27)

$$
\alpha_{n}(t) \geq \beta_{n}(t)
$$

and inequality (24) becomes

$$
\begin{aligned}
\|\mathbf{z}(\cdot, t)\|^{2} \leq & \|\mathbf{a}(\cdot)-\mathbf{w}(\cdot, 0)\|^{2} \exp \left\{-\int_{0}^{t} \beta_{n}(\tau) d \tau\right\} \\
& +\int_{0}^{t}\left(\beta_{n}(\eta)\right)^{-3}\left\|\mathbf{f}_{\eta}(\cdot, \eta)\right\|^{2} \exp \left\{-\int_{\eta}^{t} \beta_{\eta}(\tau) d \tau\right\} d \eta .
\end{aligned}
$$

This concludes the proof of the portion (13) of the theorem. For

$$
\beta_{n}(t) \geq \beta>0
$$

the inequality (13) reduces to

$$
\begin{aligned}
\|\mathbf{z}(\cdot, t)\|^{2} \leq & \|\mathbf{a}(\cdot)-\mathbf{w}(\cdot, 0)\|^{2} \exp \{-\beta t\} \\
& +\beta^{-4}(1-\exp \{-\beta t\}) \sup _{0 \leq \eta \leq t}\left\|\mathbf{f}_{\eta}(\cdot, \eta)\right\|^{2} .
\end{aligned}
$$

We see that (42) holds for

$$
\sup _{0 \leq t \leq T}\|\mathbf{f}(\cdot, t)\| \leq c_{n}^{-1} \nu^{2} \mu_{1}^{-1 / 2}\left(1-\frac{\beta}{\mu_{1} \nu}\right), \quad 0<\beta<\mu_{1} \nu
$$

which concludes the proof of the theorem.

\section{Concluding remarks}

In this section we make some observations regarding the theorem of Section 2. The main hypothesis (12) of the theorem is essentially a condition, $P<1$, on the dimensionless parameter

$$
P \equiv\left(c_{n}\|\mathbf{f}\| /\left(\mu_{1}^{1 / 2} \nu^{2}\right)\right)^{1 / 2} .
$$

Thus, the theorem is valid if the fluid is sufficiently viscous, the forcing term is sufficiently small or the flow region has sufficiently small diameter (so that $\mu_{1}$ is large). 
From the conclusions (13) or (15) of the theorem, one sees that the error $\mathbf{z}(x, t)$, between the solution of the exact problem (1) and that of the quasistationary problem (2), has small norm if $\mathbf{w}(x, 0)$ is close to $\mathbf{a}(x)$ and $\mathbf{f}(x, t)$ is slowly varying with time. Note that $\|\mathbf{f}(\cdot, t)\|$ need not be small, but need merely obey the restriction $P<1$ of the previous remark.

Thus, we have demonstrated that the quasi-steady-state approximation yields useful information for a reasonable class of problems. Although the ideas were developed here for the problem (1), it appears likely that the quasi-steady-state approach will also prove valid for a variety of problems related to (1), such as those encountered in [1], and [2].

\section{Acknowledgement}

This research was supported in part by the National Science Foundation, Grant Number MCS 821-7053.

\section{References}

[1] J. R. Cannon, E. DiBenedetto and G. H. Knightly, "The steady state Stefan problem with convection", Arch. Rational Mech. Anal. 73 (1980), 79-97.

[2] J. R. Cannon, E. DiBenedetto and G. H. Knightly, "The bidimensional Stefan problem with convection: the time dependent case", Comm. Partial Differential Equations 8 (1983), 1549-1604.

[3] D. D. Joseph, Stability of Fluid Motions II (Springer-Verlag, New York, 1976).

[4] O. A. Ladyzhenskaya, The Mathematical Theory of Viscous Incompressible Flow, 2nd Edition (Gordon and Breach, New York, 1969).

[5] M. Shinbrot, Lectures on Fluid Mechanics (Gordon and Breach, New York, 1973).

[6] R. Temam, Navier-Stokes Equations (North-Holland, New York, 1977). 